\title{
INVESTIGACIONES
}

\section{Proyecto integrador y actitudes: una perspectiva hermenéutica del desarrollo de la competencia en la docencia}

\author{
Integrative project and attitudes: a hermeneutical perspective of the development \\ of competence in teaching
}
Projeto integrativo e atitudes: uma perspectiva hermenêutica do desenvolvimento da competência docente

\author{
P. M. S. Silva ${ }^{a}$, C. S. Pires ${ }^{a, b}$, M. A. A. Pimenta ${ }^{a}$ \\ ${ }^{a}$ Estudiante de maestría en Universidad de Sorocaba - \\ UNISO y profesora de la Facultad de Tecnología (FATEC), Brasil. \\ paty_mac_sampaio@hotmail.com, maria.pimenta@prof.uniso.br \\ ${ }^{b}$ Estudiante de maestría en Universidad de Sorocaba - \\ UNISO y profesora del Instituto Federal de Educación, Ciencia y Tecnología de São Paulo (IFSP), Brasil. \\ cristiane.sales.pires@gmail.com,
} \begin{abstract}
para el trabajo, la inserción de los trabajadores en el mercado y la mejora de su desempeño profesional. Sin embargo, el papel de la escuela debe ser más amplio para garantizar la formación integral de los estudiantes. Este artículo destaca el potencial de la práctica pedagógica de la Educación Profesional, en el sentido de desarrollar más que el contenido técnico que subyace en los conocimientos y habilidades, los valores y actitudes que determinan el comportamiento del futuro profesional. La investigación realizada a partir de la pesquisa bibliográfica y el análisis documental tiene como objetivo demostrar cómo la propuesta de los Proyectos Integradores (PI) puede contribuir a la formación de competencias, incluyendo las dimensiones del conocimiento (saber), habilidades (saber hacer) y actitudes (saber ser), siendo este último, el más frágil; es decir, el menos trabajado entre las otras dimensiones. Como componente curricular ancla de las disciplinas profesionales, el Proyecto Integrador (PI) promueve la interdisciplinariedad, se corrobora con el desarrollo de contenidos técnicos específicos y brinda oportunidades para el desarrollo de valores y actitudes, cumpliendo así la función de la escuela, que es garantizar la formación integral de los estudiantes.

Palabras claves: Enseñanza. Educación profesional. Proyecto integrador. Competencia. Dimensión actitudinal.
\end{abstract}

En Brasil, la Educación Profesional y Tecnológica apunta a la calificación profesional cuyo objetivo es la formación

\section{ABSTRACT}

Professional and Technological Education aims at professional qualification whose objective is to prepare and insert the students in the labor market and improve their professional performance. However, the role of school must be broader in order to guarantee the students integral formation. This article highlights the potential of the pedagogical practice of Professional Education in the sense of developing more than the technical contents that underlie within it, the knowledge and skills, the values and attitudes which are decisive factors of the future professional behavior. The investigation was carried out based on bibliographic research and documentary analysis and intends to demonstrate how the proposal of Integrating Projects (IP) can contribute to formation of competence, including the dimensions of knowledge, skills (knowing how to do) and attitudes (knowing how to be), being this last one, the most fragile, and the least commonly worked in comparison to the other 
dimensions. As the anchor curricular component of professional disciplines, the Integrator Project (IP) promotes interdisciplinary nature, corroborates with development of specific technical contents and provides opportunities to develop values and attitudes, thus fulfilling the role of school which is to guarantee students integral formation.

Key words: Schooling, Professional Education, Integrator Project, Competence, Attitudinal dimensión.

\section{RESUMO}

No Brasil, a Educação Profissional e Tecnológica visa à qualificação profissional cujo objetivo é a formação para o trabalho, inserção do trabalhador ao mercado e a melhoria de desempenho profissional. No entanto, a função da escola deve ser mais ampla no sentido de garantir a formação integral dos estudantes. Este artigo destaca o potencial da prática pedagógica da Educação Profissional, no sentido de desenvolver mais que os conteúdos técnicos que fundamentam os conhecimentos e habilidades, os valores e atitudes determinantes no comportamento do futuro profissional. A investigação realizada a partir de pesquisa bibliográfica e análise documental pretende demonstrar como a proposta dos Projetos Integradores (PI) pode contribuir na formação da competência incluindo as dimensões do conhecimento (saber), habilidades (saber fazer) e atitudes (saber ser), sendo esta última, a mais frágil, ou seja, a menos comumente trabalhada dentre as demais dimensões. Como componente curricular âncora das disciplinas profissionalizantes, o Projeto Integrador (PI) promove a interdisciplinaridade, corrobora com desenvolvimento de conteúdos técnicos específicos, e proporciona oportunidade de desenvolvimento de valores e atitudes, atendendo, desta forma, a função da escola que é garantir a formação integral dos estudantes.

Palavras-chave: Educação Escolar, Educação Profissional, Projeto Integrador, Competência, Dimensão Atitudinal.

\section{INTRODUCCIÓN}

El mantenimiento de la vida humana exige esfuerzo que pasa por el trabajo. Actuar sobre la naturaleza para transformarla según sus necesidades es obra del hombre para producir condiciones de bienestar. Por tanto, se puede decir que la esencia del hombre es el trabajo, que a su vez, no nace listo, se hace humano a lo largo de la vida en sus vivencias e interacciones con otros sujetos. En la historia de la educación hay un fuerte llamado a la preparación técnica, descuidando el desarrollo de virtudes y valores: ¿fracasó la escuela en su función de formación humana?

La modalidad docente en Brasil que prepara para el mercado laboral es la Educación Profesional y Tecnológica, apoyada por políticas públicas desde 1930 e instituida en 1937 por la Constitución Federal, en su Art. 129. El Proyecto Integrador, práctica pedagógica común de esta modalidad de educación, es una estrategia interdisciplinaria que propone la resolución de un problema práctico a través de la investigación a realizarse en etapas que son guiadas por un docente que asegura la integración, articulación y aplicación de los conocimientos de los componentes curriculares de ese período escolar. Es a través de la investigación que la visión general del alumno se completa durante su camino formativo y sienta bases sólidas en la construcción de competencias.

Al demostrar cómo la implementación de Proyectos Integradores (PIs) puede contribuir al desarrollo de la Dimensión Actitudinal de la Competencia en el trabajo en grupo, este artículo pretende, en general, resaltar el potencial de esta práctica pedagógica en la Educación Profesional, además del contenido técnico, proporcionando oportunidades prácticas para ejercitar virtudes, valores y actitudes ${ }^{1}$ que son determinantes en el comportamiento del futuro profesional. Al buscar soluciones a problemas reales, plantear hipótesis, sistematizar

Estas palabras, del mismo campo semántico, indican características frente a la vida que ayudan a abordar cuestiones morales y éticas. 
estudios, planificar tiempos y recursos, la necesidad de identificar recursos internos como paciencia, liderazgo, disciplina, determinación que se deben movilizar en la conclusión y presentación del proyecto a un panel de docentes e invitados. De esta forma, se estimula el reconocimiento y la reflexión sobre las virtudes, valores y actitudes como factores rectores (Bennet, 1995; Comte-Sponville, 1999) de conductas que viabilizan el proyecto.

La investigación se justifica resaltando la importancia de los Proyectos Integradores en el desarrollo de la Dimensión Actitudinal, hasta entonces desatendida en los planes de estudio de la Educación Profesional, que siempre ha sido relevante para la formación humanística y que actualmente es requerida por la sociedad contemporánea y, en consecuencia, por el mercado de trabajo.

Las bases teóricas para la discusión se presentan de forma secuencial: (i) Relación escuela y trabajo, (ii) Educación profesional y tecnológica y su relación con la sociedad, (iii) Concepto de competencia para la educación y la evolución en el tiempo e interrelación con el mercado laboral, se discute la importancia de (iv) la Dimensión actitudinal. Luego de una breve síntesis sobre la metodología adoptada, la sección de Resultados presenta el Proyecto Integrador como una práctica pedagógica para el desarrollo de Competencias.

En la Sección de Discusión, los temas están relacionados. En conclusión, se señala la complejidad de implementar el Proyecto Integrador en vista de las especificidades de la Educación Profesional.

\section{LAS BASES TEÓRICAS PARA PENSAR EN EL TEMA}

\subsection{RELACIÓN ENTRE EDUCACIÓN Y TRABAJO}

El proceso educativo entrelaza la producción del hombre (trabajo) y la formación humana. La palabra institución proviene del latín institutio y tiene varios significados: ordenamiento, educación, creación, formación, método, sistema, escuela, entre otros. La idea de algo que no está terminado y que se reconfigura continuamente. El proceso de institucionalización de la educación coincide con el surgimiento de la sociedad de clases y la profundización de la división del trabajo. En las sociedades primitivas, la educación era una acción espontánea, común a todos los miembros y se producía al mismo tiempo que la actividad laboral. Posteriormente, la división de clases dio lugar a otra división: la de la educación. Este lugar, destinado a la clase dominante, pasó a denominarse escuela, que en griego significa "lugar de esparcimiento", atribuido a quienes tenían tiempo libre para el esparcimiento mientras los demás continuaban siendo instruidos en el lugar de trabajo. Desde la antigüedad hasta nuestros días, la institución escolar se ha ido perfeccionando y haciéndose cada vez más compleja, sirviendo siempre como parámetro y referencia a todas las formas de educación. Sin embargo, esto no significa que haya mantenido la misma calidad a lo largo del tiempo, pero ciertamente se ha expandido enormemente en términos cuantitativos (Saviani, 2005, 2007). De esta relación surge la institución educativa.

Esa es la historia y función de la escuela: mutante e inacabada, a veces orientada al ocio de los más privilegiados, a veces atendiendo a la calificación profesional de los menos privilegiados; a veces abordando valores y actitudes de dogmas religiosos, a veces desconcertado al lidiar con cuestiones socioemocionales como la ética, los valores y las actitudes (Saviani, 2005, 2007; Goergen, 2005). 
El trabajo, a su vez, siempre ha sufrido metamorfosis adaptándose al modo de producción actual. Palabras como informatización, informalización, precariedad, flexibilidad, empleabilidad componen las discusiones sobre los efectos de la profundización del proceso de globalización en las relaciones laborales. Sin embargo, una necesidad se mantiene vigente desde hace al menos dos siglos: la construcción de Competencias.

Los estudios científicos de Administración y práctica empresarial asumen la Competencia como la capacidad de articular y aplicar conjuntamente conocimientos, habilidades y actitudes, concepto que subyace en la teoría CHA (McClelland, 1973; Durand, 2000). Si bien el campo de la Educación asume el concepto de competencia como la capacidad de movilizar y articular recursos personales para la solución de un problema complejo (Boterf, 2003; Perrenoud, 1999), se encontró que la teoría CHA se basaba en investigaciones e intervenciones prácticas del educador y pedagogo Pestalozzi. Este hallazgo ha sido confirmado y perfeccionado en las últimas décadas por estudiosos de diferentes campos del conocimiento como Bloom, y Perrenoud, Durand. En el mercado laboral, el concepto de Competencia, basado en la teoría CHA, se devuelve al campo educativo con un mensaje inquietante: los egresados del sistema educativo son contratados por aspectos técnicos (conocimientos y habilidades) pero son despedidos por temas relacionados con la ética, comportamiento, virtudes y valores (actitudes).

\subsection{LA EDUCACIÓN PROFESIONAL Y TECNOLÓGICA Y SU RELACIÓN CON LA SOCIEDAD}

Históricamente, el proceso educativo incluyó la formación integral del individuo de acuerdo a su clase social: la educación de la élite altamente personalizada, los tutores orientaron el desarrollo de la mente del individuo, principio básico de habilidades y talentos. Para quienes no pertenecían a la clase privilegiada, la formación era un asunto personal, gestionado por familias y comunidades locales (Torres y Burbules, 2004).

En la construcción de una sociedad moderna, la Educación Profesional y Tecnológica ganó fuerza, convirtiéndose en una modalidad de enseñanza reconocida oficialmente en varios países. En el Brasil imperial, la formación de la fuerza laboral fue, en un principio, responsabilidad de asociaciones religiosas y filantrópicas. Sin embargo, la inquietud por brindar una formación obligatoria a los trabajadores en diversos oficios, combinada con el objetivo de sacar a los niños de la calle para que no se conviertan en futuros desempleados, hizo que el gobierno imperial derivara a los huérfanos a las Empresas de Aprendices de Artesanos y a la Empresas de Aprendices de Marineros, que forman el contingente profesional de actuación en la marina y en la guerra. Dichas instituciones se mantuvieron en algunos estados después de la Proclamación de la República y se convirtieron en la base para el desarrollo de las redes de escuelas vocacionales brasileñas, las llamadas Escuelas Artísticas de Aprendices creadas en 1909 (García, 2018). Desde 1930, la Educación Profesional se ha incorporado a las políticas públicas nacionales, desarrollándose desde distintos dispositivos legales. El Cuadro 1 presenta las acciones gubernamentales en Educación Profesional en Brasil. 
Tabla 1. Dispositivos Legales de la Educación Profesional Brasileña

\begin{tabular}{|c|c|}
\hline Año & Dispositivos legales \\
\hline 1909 & El Decreto No 7.566 crea las Escuelas de Aprendices y Artesanos. \\
\hline \multirow{2}{*}{1937} & $\begin{array}{l}\text { La Constitución Federal se ocupa de la educación profesional e industrial en su artículo } \\
129 .\end{array}$ \\
\hline & $\begin{array}{l}\text { La Ley No. } 378 \text { transforma las escuelas de aprendices y artesanos mantenidas por la } \\
\text { Unión en escuelas secundarias e industriales. }\end{array}$ \\
\hline 1942 & El Decreto Ley $N^{\circ} 4.048$ crea el SENAI (Servicio Nacional de Aprendizaje Industrial). \\
\hline 1946 & $\begin{array}{l}\text { El Decreto Ley No. } 8.621 \text { crea el Servicio Nacional de Aprendizaje Comercial - Senac, y } \\
\text { se reglamenta el aprendizaje de aprendices. }\end{array}$ \\
\hline 1959 & $\begin{array}{l}\text { Las escuelas técnicas federales se establecieron como municipios, basadas en escuelas } \\
\text { industriales y técnicas mantenidas por el Gobierno Federal. }\end{array}$ \\
\hline 1961 & $\begin{array}{l}\text { La Ley } \mathrm{N}^{\circ} 4.024 / 61 \text { permite a los egresados de cursos de formación profesional } \\
\text { continuar sus estudios en la educación superior ( } 1^{\mathrm{a}} \text { versión LBD). }\end{array}$ \\
\hline 1968 & $\begin{array}{l}\text { La Ley } \mathrm{N}^{\circ} 5.540 \text { permite la oferta de cursos de educación superior para la formación de } \\
\text { Tecnólogos. }\end{array}$ \\
\hline 1971 & $\begin{array}{l}\text { La Ley n }{ }^{\circ} 5.692 \text { define que toda educación secundaria, ahora denominada bachillerato, } \\
\text { debe conducir a la conclusión de un título profesional técnico o, al menos, de asistente } \\
\text { técnico mediante un título parcial. }\end{array}$ \\
\hline 1991 & $\begin{array}{l}\text { La Ley } N^{\circ} 8.315 \text { crea el Servicio Nacional de Aprendizaje Rural (Senar), en un formato } \\
\text { institucional similar al del Senai y Senac. }\end{array}$ \\
\hline 1996 & $\begin{array}{l}\text { Se promulgó la segunda Ley de Bases y Lineamientos para la Educación Nacional } \\
\text { (LDB), que dedica el Capítulo III de su Título VI a la educación profesional. } \\
\text { Posteriormente, este capítulo fue denominado "De Educación Profesional y Tecnológica" } \\
\text { por la Ley } \mathrm{N}^{\circ} 11.741 \text { / } 2008 \text {, que incluyó la fracción IV-A en el Capítulo II, para abordar } \\
\text { específicamente la educación técnica profesional de alto nivel. }\end{array}$ \\
\hline 1998 & $\begin{array}{l}\text { La Resolución CNE/CEB No. 04/99 y la Opinión CNE/CEB No. 16/99 definen los } \\
\text { Lineamientos Curriculares Nacionales para la Educación Profesional a Nivel Técnico }\end{array}$ \\
\hline 2002 & $\begin{array}{l}\text { La Resolución CNE/CP N }{ }^{\circ} 03 / 02 \text { y la Opinión CNE/CP N }{ }^{\circ} 29 / 02 \text { definen los } \\
\text { Lineamientos Curriculares Nacionales para la Formación Profesional a Nivel } \\
\text { Tecnológico. }\end{array}$ \\
\hline 2005 & $\begin{array}{l}\text { La Resolución CNE/CEB nº 1/05, y el Dictamen CNE/CEB n } 39 / 04 \text {, actualizan los } \\
\text { Lineamientos Curriculares Nacionales para la Formación Profesional a Nivel Técnico. }\end{array}$ \\
\hline \multirow[b]{2}{*}{2008} & $\begin{array}{l}\text { La Resolución CNE / CEB no 3/08 y el Dictamen CNE/CEB n }{ }^{\circ} 11 / 08 \text {, regula la } \\
\text { institución y la implantación del Catálogo Nacional de Cursos Técnicos de Nivel Medio } \\
\text { - CNCT en las redes públicas y privadas de Educación Profesional. }\end{array}$ \\
\hline & $\begin{array}{l}\text { La Ley } 11.741 \text { introduce modificaciones al Capítulo III, Título V de la LDB, que ahora } \\
\text { trata de "La Educación Profesional y Tecnológica", así como también introduce una } \\
\text { nueva Sección en el Capítulo II del mismo título, la sección IV-A, cuarta "de Educación } \\
\text { Profesional. Técnica de nivel medio". }\end{array}$ \\
\hline
\end{tabular}




\begin{tabular}{|c|l|}
\hline 2012 & $\begin{array}{l}\text { La Resolución CNE/CEB n }{ }^{\circ} \text { 6/12 y el Dictamen CNE/CEB n }{ }^{\circ} \text { 11/12 actualizan los } \\
\text { Lineamientos Curriculares Nacionales de la Educación Técnica Profesional de Alto Nivel. }\end{array}$ \\
\hline 2014 & $\begin{array}{l}\text { La Ley n } n^{\circ} 13.005 / 14 \text { aprueba el nuevo Plan Nacional de Educación que prevé "ofrecer, al } \\
\text { menos, el 25\% (veinticinco por ciento) de la matrícula de educación de jóvenes y adultos } \\
\text { en educación primaria y secundaria, de manera integrada con la formación profesional", } \\
\text { así como "triplicar la matrícula en educación secundaria técnica y profesional, } \\
\text { asegurando la calidad de la oferta y al menos el 50\% (cincuenta por ciento) de la } \\
\text { expansión en el segmento público". }\end{array}$ \\
\hline 2017 & $\begin{array}{l}\text { La Ley no 13.415/07 modifica la LDB, incluyendo el itinerario formativo "Formación } \\
\text { Técnica y Profesional" en el bachillerato, que prevé la inclusión de experiencias laborales } \\
\text { prácticas ya sea en el sector productivo o en entornos de simulación, así como el } \\
\text { otorgamiento de certificados intermedios de calificación. }\end{array}$ \\
\hline
\end{tabular}

Fuente: Adaptado de BRASIL, Ministerio de Educación [s.f.] (http://portal.mec.gov.br).

Se nota en el historial presentado en ese cuadro que la Educación Profesional es relevante para la sociedad y el desarrollo de la nación; y mientras se incorporan nuevos dispositivos a las políticas públicas nacionales, se modifican y actualizan la preparación profesional, la cual está ligada al desarrollo económico nacional.

El proceso de institucionalización de la educación, descrito en la introducción de este artículo, coincide con el surgimiento de la división de clases; desde entonces, la escuela ha sufrido varias transformaciones, pero siempre ha traído consigo la expectativa de la sociedad que espera que se cumpla la función de preparar al estudiante para la vida y el trabajo (Saviani, 2005).

La actual profundización de la globalización y la constante metamorfosis del modo de producción han adelantado ciertas tesis sobre la fuerte reducción de la clase obrera en los próximos años.

Incapaz de juzgar si tales predicciones se harán realidad o no, Antunes (2014) prefiere apuntar en el horizonte una nueva morfología del trabajo caracterizada por la informatización del infoproletariado o cibertariato (expansión del call center y telemarketing) y el aumento de la informalización bajo la forma de trabajadores subcontratados, subcontratistas, flexibles, a tiempo parcial, teletrabajadores, ampliando el universo del trabajo precario.

\subsection{CONCEPTO DE COMPETENCIA EN EDUCACIÓN}

El concepto de competencia aceptado en el campo de la Educación es la capacidad de movilizar recursos dentro de un contexto para resolver situaciones complejas, impredecibles, cambiantes y siempre singulares (Boterf, 2003; Perrenoud, 1999). Sin embargo, al mirar la evolución histórica de este concepto, se observa que en el siglo XVIII Johann Heinrich Pestalozzi reconoció tres claves del aprendizaje individual, llamándolas Cabeza, Manos y Corazón. Dos siglos después, Bloom y su equipo, en un amplio estudio, elaboraron la taxonomía de los objetivos educativos, contemplando la tríada en dominios, llamándolos cognitivos, psicomotores y afectivos. Más recientemente, en el año 2000, la esencia de estos conceptos clave es retomada por Durand, quien reconstruye el marco de competencias a partir de investigaciones surgidas del campo de la Educación y presenta 
un modelo dinámico de construcción de competencias en su artículo "La Alquimia de la Competencia", entrelazando finalmente las relaciones interdependientes entre aprendizaje y competencia (Freitas y Brandão, 2005). La evolución de este concepto está representada en la Figura 1:

Figura 1. Las relaciones conceptuales entre aprendizaje y competencia

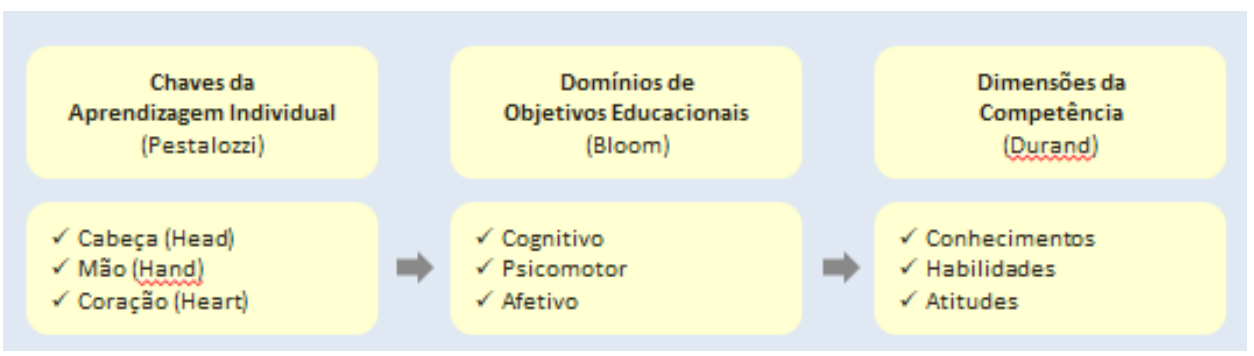

Fuente: Brandão, Guimarães y Borges-Andrade (2001), citados en Freitas e Brandão (2005, p. 4).

De esta forma, la competencia puede entenderse como el resultado de un flujo continuo de aprendizaje que refuerza y amplía la base competencial inicial (Durand, 2000).

\subsection{LA DIMENSIÓN ACTITUDINAL}

Existen variadas definiciones sobre competencia en la literatura especializada. Entre ellos, destacan dos tendencias: la norteamericana y la francesa, esta última asumiendo una perspectiva más desarrollada de la competencia. Es decir, la combinación de conocimientos (saber) y formas de actuar (habilidad) se basaría en actitudes positivas que favorezcan la movilización de recursos personales para una determinada actuación.

Entre estas actitudes, la búsqueda activa de nuevos aprendizajes minimiza las posibles limitaciones en las otras dimensiones (conocimiento y habilidad), (Gondim et al., 2014). Durand (2000) representante de la línea de investigación francesa, reafirma la importancia de la dimensión actitudinal en la movilización de conocimientos (saberes) y habilidades (acciones), porque las actitudes están relacionadas con el comportamiento, la identidad y la voluntad; y estos están influenciados por un marco de valores y virtudes o la falta de ellos, para orientar y brindar seguridad. Esta relación con virtudes y valores también puede explicar la frecuencia con la que se atribuye la actitud al sentido de proactividad. Por ejemplo, a nivel individual, el conocimiento sin una actitud adecuada puede volverse estéril mientras que una actitud sin conocimiento es ineficaz. A nivel colectivo, ¿qué sería del conocimiento grupal sin las actitudes adecuadas en las relaciones intergrupales? ¿Qué sucede cuando un miembro no sabe cómo comportarse en un grupo? ¿O cuando el propio grupo no sabe cómo comportarse como grupo?

El dominio de la competencia se puede adquirir a lo largo del proceso de socialización familiar, educativa y profesional, en el que se articulan las tres dimensiones: conocimiento, habilidad y actitud. El aprendizaje juega un papel importante en este proceso y puede ser 
tanto informal como formal, con instituciones educativas a cargo del aprendizaje formal, que se basan en métodos, técnicas y estrategias diseñadas deliberadamente para generar cambios (Gondim et al., 2014). Sin embargo, desde la Edad Moderna, cuando la educación religiosa ya no es un referente de las normas morales, la escuela o la educación ha carecido de una norma en la forma de abordar valores o formas de comportamiento. Así, el tema de la ética ocupa predominantemente el lugar de la transversalidad. No hay espacio ni tiempo para discutir y desarrollar valores que regulen el comportamiento. Hay falta de reflexión sobre el equilibrio entre la cooperación pacífica y la autoafirmación personal, contextualizando la conducta individual y el bienestar colectivo. Poco se discute sobre el consenso de principios que pueden servir de guía para las elecciones y comportamientos éticos de las personas que aspiran a una vida más digna, respetuosa y solidaria en una sociedad justa y democrática (Goergen, 2005).

\section{METODOLOGÍA}

Esta investigación sigue el enfoque cualitativo y descriptivo, que como señala Gil (2002) aborda la descripción de las características de un fenómeno dado, considerando el proyecto integrador como una práctica pedagógica que permite contribuir a la formación de competencias, incluyendo las dimensiones del conocimiento (saber), habilidades (saber hacer) y actitudes (saber ser), con énfasis en esta última, que en general es la menos trabajada entre las otras dimensiones, dadas las dificultades de medición y su inmediata invisibilidad.

El enfoque metodológico utilizado fue el fenomenológico-hermenéutico que para Gamboa (1998) se traduce en una reflexión creativa del autor para explicar lo implícito, en la que el texto se explica por el contexto, y el significado se encuentra en el contexto universal en el que se inserta. Teniendo al hombre como un ser de relaciones, este enfoque busca sistematizar metodológicamente una experiencia.

Para los procedimientos técnicos, se decidió utilizar la investigación bibliográfica y documental, con el fin de conocer la realidad y construir con criterio y cientificidad, una confluencia entre la Educación Profesional y la Dimensión Actitudinal en la formación de competencias (Lakatos y Marconi, 1992).

\section{RESULTADOS}

Es innegable que el trabajo aporta dignidad social y económica a los hombres, ya que su exclusión prácticamente los deja al margen de la vida social y cultural. Sin embargo, la Educación Profesional debe preocuparse no solo por la preparación para el trabajo, sino también por la plena realización del ser humano (Streck y Viola, 2014).

Sin duda, los fenómenos contemporáneos afectan mucho al empleo y al objetivo de la educación en términos de preparación para el trabajo. A la competitividad del trabajo internacional se suma la demanda de nuevas habilidades y actitudes necesarias para adaptar las actividades laborales o, incluso, a los diversos cambios de función a lo largo de la vida. Estos mercados inestables deberían guiar la misión de las instituciones escolares contemporáneas (Torres y Burbules, 2004). 
En este contexto, la Educación Profesional, primera representante de la docencia vinculada al trabajo, no solo debe ceñirse al puesto de trabajo, sino preparar al alumno para vivir en esta sociedad altamente especializada y competitiva. Saviani (2007) señala algunas características necesarias para esta modalidad educativa: (1) Ser tecnológica, en el sentido de unir trabajo-estudio o formación intelectual-trabajo productivo. (2) Ser multiprofesional; o sea, no restringido a la división del trabajo. (3) Apropiarse del concepto de omnilateralidad, atributo del hombre como ser integral como posibilidad de manifestarse independientemente de las posiciones específicas que ocupe en el trabajo, la cual se opone a la unilateralidad que propone la educación que apunta únicamente a la formación para el trabajo (Saviani, 2007).

La Educación Profesional es el locus privilegiado donde tiene lugar la formación de competencias. Sin embargo, a pesar del esfuerzo en la preparación de su contingente de estudiantes, una jerga muy conocida en el mundo empresarial se repite entre los pasillos de las organizaciones: se contrata profesionales por aspectos técnicos (conocimientos y habilidades); y en su mayor parte, se descartan por problemas de conducta. (Borges, 2012). Los resultados de estas encuestas corroboran la percepción de los profesionales de recursos humanos, evidenciando así la demanda del mercado laboral relacionada con la Dimensión Actitudinal en la formación de competencias de los egresados de la educación profesional y tecnológica.

\subsection{PROYECTO INTEGRADOR COMO PRÁCTICA PEDAGÓGICA PARA EL DESARROLLO DE COMPETENCIAS}

La práctica pedagógica es una acción intencionada, consciente y participativa del docente con el fin de satisfacer determinadas expectativas en relación con el acto de educar. La práctica pedagógica va desde la planificación y sistematización de los procesos de aprendizaje hasta la ejecución de estos, y debe considerar los espacios y tiempos de la escuela, las opciones de organización del trabajo y las posibles alianzas. Cuando un maestro comprende el propósito de la clase y busca integrarlo en un contexto más amplio de la educación del alumno, está contribuyendo al proceso educativo. Si bien la práctica pedagógica comienza en la persona del docente, se extiende más allá de él, involucrando a los estudiantes y a la comunidad en la que se inserta la escuela. Esto se debe a que se basa en pactos sociales, negociaciones y decisiones colectivas, que cumplen con la intencionalidad del proceso educativo, incorporando reflexiones continuas y colectivas (Franco, 2016).

Moura (2007) apunta al Proyecto Integrador (PI) como una alternativa de práctica pedagógica al servicio de la formación integrada e integral del alumno. Los PIs tienen como objetivo movilizar e interrelacionar los conocimientos desarrollados por los sujetos de un período académico en la búsqueda de la solución de un problema determinado. Los conocimientos parciales y específicos de cada disciplina se entrelazan hasta que componen un conocimiento más amplio y completo. La contextualización del problema propuesto a los estudiantes sirve como eje integrador de los componentes curriculares, da sentido al aprendizaje, da vida a la relación del contenido teórico y a la realidad vivida. Al hacer investigación, los estudiantes construyen autonomía intelectual, además de desarrollar actitudes de ciudadanía, solidaridad y responsabilidad social.

En general, el Proyecto Integrador tiene como objetivo estimular la investigación desarrollando la capacidad de toma de decisiones, planificación, gestión del tiempo, 
desarrollo del sentido crítico, entre otros. En el momento de las entregas finales del semestre académico, se contempla el desarrollo de la oralidad y la adopción de escritura estandarizada. Los procedimientos de los PIs buscan resolver un problema complejo para ser resuelto en equipo, estimulando así la construcción de habilidades profesionales e interpersonales a través de interacciones entre los miembros del grupo (Santos y Barra, 2012).

En la dinámica entre los campos teóricos y prácticos, el alumno va mejorando su proceso de socialización, dando sentido al mundo que lo rodea mientras aprende a ejercitar su singularidad ante el grupo y reconocer la del otro, confluyendo con el triple proceso educativo, concebido por Charlot: humanización-socialización-subjetivación/singularización.

\section{DISCUSIÓN}

El Proyecto Integrador (PI) establece un punto de anclaje común entre las asignaturas del período académico. Se configura como un eje integrador y catalizador que articula diversas áreas de conocimiento y contenidos formativos. Además de la interdisciplinariedad, induce la transversalidad porque relaciona el aprendizaje de conocimientos teóricamente sistematizados con cuestiones de la vida real (Santos y Barra, 2012). Al entrelazar el conocimiento de las disciplinas en la solución de problemas reales, el PI contribuye a la construcción de la Competencia en el aspecto de la Dimensión del Conocimiento (saber).

La estrategia de problematización del PI es una dinámica empoderadora que desafía al estudiante a movilizar y aplicar conocimientos de diferentes áreas del saber, estimulando la capacidad de analizar su entorno, contextualizando el conocimiento con la realidad, combinando y aplicando teoría y práctica profesional, (Moura, 2007; Santos y Barra, 2012) corroborando la Dimensión de la Habilidad (saber hacer).

La investigación como principio educativo Proyecto Integrador involucra los procesos de delimitación de un tema, planteamiento de hipótesis, planificación de etapas de actividades, recolección de datos, elaboración del estudio bibliográfico, confirmación o no de hipótesis, tratamiento de datos, elaboración de trabajos escritos en estructura académica, preparación para presentar los resultados y defender públicamente el proyecto ante un panel de profesores e invitados. Como ejercicio colectivo, el proceso fomenta la adopción de actitudes por parte de los integrantes del grupo que sean consistentes con la ejecución de las tareas, el ejercicio del liderazgo, la disciplina, la capacidad de trabajar en grupo y el cumplimiento de los plazos (Moura, 2007; Santos y Barra, 2012). Todas estas situaciones estimulan la madurez de la dimensión actitudinal del alumno.

La ciencia como guía para el PI les brinda a los estudiantes una mayor familiaridad con el proceso de investigación. En la versión de investigación aplicada, orientada a la producción de bienes y servicios, despierta la criticidad del estudiante por resultados que puedan mejorar las condiciones de vida colectiva. En la investigación orientada a aspectos más académicos relacionados con las ciencias naturales y las ciencias sociales, conduce a comprender diferentes intereses e identificar a los probables beneficiarios de los posibles resultados alcanzados. En todo caso, el Proyecto Integrador contempla la construcción, deconstrucción y reconstrucción de sus propias interpretaciones de la ciencia, en la capacidad de investigación para mejorar las condiciones de la vida colectiva, el mundo y la vida misma, cumpliendo así con la premisa básica de la institución escolar que es la formación integral del alumno (Moura, 2007; Santos y Barra, 2012). 


\section{CONSIDERACIONES FINALES}

El Proyecto Integrador trae al aula una oportunidad única para abordar sistemáticamente la Dimensión Actitudinal y su importancia en la Educación Profesional y Tecnológica. Sin embargo, en la práctica surgen dificultades que frustran a profesores y estudiantes.

Los docentes de los PIs, herederos de una formación deficiente en términos de vocabulario y estrategias de comportamiento y actitudes, están obligados a realizar competencias en temas como manejo de conflictos intra y/o intergrupales, manejo de emociones de los estudiantes, regulación de la conducta, acceso a la motivación movilizadora, instigar en el alumno la transición entre los roles de líder y liderado, entre otros. A menudo, no hay recursos personales a menos que este profesional haya tenido modelos inspiradores en la familia, en la sociedad, en su círculo de amigos para este ejercicio profesional y personal.

El profesor es, además, heredero de un sistema educativo altamente especializado y fragmentado, proveniente de una sociedad de producción fordista y posfordista, cuyas cuestiones de comportamiento y actitudes son manejadas por profesionales como psicólogos, psiquiatras y terapeutas. Sin embargo, los profesores del PI son pedagogos, administradores, sociólogos, biólogos, etc. Este es el resultado de lamentaciones como "No estudié para esto", "No aprendí en la escuela de posgrado a dejar de lado las peleas de los estudiantes".

La tercera herencia proviene de la propia tradición de la educación como transmisión de conocimientos. Aquí la metáfora de la línea de producción sirve bien: numerosas pautas para Proyectos Integradores se paralizan porque los grupos no se entienden, como si la Dimensión Actitudinal fuera una pieza defectuosa que no debería pasar.

Otro tema que surge es la dificultad de implementar el trabajo colaborativo que requiere este tipo de propuestas, por lo que el docente del PI muchas veces trabaja sin la colaboración de compañeros de trabajo. Al pedir sugerencias a los profesores para proponer problemas de investigación, a medida que se desarrolla el módulo, se busca integrar conocimientos fragmentados en un eje, en general, el proceso para, en las disciplinas de los compañeros. No existe un modelo colaborativo por parte de los docentes, lo que pone de manifiesto la complejidad de desarrollar la interdisciplinariedad: ¿son todos agentes de la interdisciplinariedad o es solo el profesor coordinador del PI el responsable de esta misión?

Otra dificultad surge del malentendido de los estudiantes sobre el proyecto, el método científico de investigar problemas, la afirmación de que el docente no comprende los contenidos de las otras materias por lo que no comprende el tema. Nuevamente, cuando el contenido se detiene por conflictos personales, la afirmación de que "hoy prácticamente no tuvimos clase en el PI porque los grupos no se llevan bien”.

La investigación detenida indica la resistencia de los estudiantes a comprender que es precisamente el conflicto, los desafíos de comportamiento y la actitud hacia el PI los que forman parte de la formación humana.

Como se comentó anteriormente, el PI es una práctica pedagógica que comienza con el docente, pero no depende solo de él. El PI se da en medio de pactos sociales, negociaciones y deliberaciones colectivas, que lo fortalecen para "preservar los tesoros de la civilización dentro del proceso de socialización, de los integrantes de cada nueva generación" (Torres y Burbules, 2004, p. 12). La falta de comprensión por parte de profesores y alumnos de que si "se detiene la producción" no es un pequeño detalle defectuoso, sino una razón fundamental para estar aquí, para el PI y para la vida. 
Además de las complejidades presentadas, hoy en día existe una gran dificultad para reestructurar el modelo y modo de funcionamiento del PI ante la reciente epidemia del Covid19, cuyo prolongado aislamiento social ha impactado a la sociedad y especialmente a las escuelas. Como reflexión final, las preguntas siguen siendo: ¿cómo mantener el compromiso social en el PI durante y después del aislamiento social prolongado? ¿Las actividades y experiencias mediadas por la tecnología de la información y la comunicación son eficientes en este proceso o mantienen a las personas separadas?

Finalmente, la debilidad del enfoque escolar respecto a la Dimensión Actitudinal puede ser una de las causas de la precariedad de los lazos sociales por el fuerte atractivo del individualismo. De esta forma, los PIs se configuran como un aporte costoso y relevante para construir la existencia humana más significativa.

\section{REFERENCIAS BIBLIOGRÁFICAS}

Antunes, R. (2014). Desenhando a nova morfologia do trabalho no Brasil. Estudos Avançados, 28(81), 39-53. https://doi.org/10.1590/S0103-40142014000200004

Bennett, W. J. (1995). O livro das virtudes: uma antologia de William J. Bennett. Nova Fronteira.

Borges, L. (2012). Contrata-se pela competência técnica e demite-se pela comportamental. Portal Administradores.com. Disponível em https://administradores.com.br/artigos/contrata-se-pelacompetencia-tecnica-e-demite-se-pela-comportamental. Acesso em 22 set. 2020.

Boterf, G. (2003). Desenvolvendo as competências profissionais. Porto Alegre: Artmed

Brasil, Ministério da Educação. [s.d] Histórico da Educação Profissional e Tecnológica no Brasil. Disponível em: http://portal.mec.gov.br/publicacoes-para-professores/30000-uncategorised/68731historico-da-educacao-profissional-e-tecnologica-no-brasil. Acesso em 11 Set. 2020.

Charlot, B. (2014). Da relação com o saber às práticas educativas [livro eletrônico]. 1. ed. Cortez.

Comte-Sponville, A. (1999). Pequeno tratado das grandes virtudes. Martins Fontes.

Durand, T. (2000). L'alchimie de la compétence. Revue Française de Gestion, Paris, 127, 84-102, Janvier-Février.

Gondim, S. M. G, Morais, F. A. \& Brantes, C. A. A. (2014). Competências socioemocionais: fatorchave no desenvolvimento de competências para o trabalho. Revista Psicologia Organizações e Trabalho, 14(4), 394-406. Recuperado em 07 de outubro de 2020, de http://pepsic.bvsalud.org/ scielo.php?script=sci_arttext\&pid=S1984-66572014000400006\&lng=pt\&tlng=pt.

Franco, M. A. R. S. (2016). Prática pedagógica e docência: um olhar a partir da epistemologia do conceito. Revista Brasileira de Estudos Pedagógicos, 97(247), 534-551. https://doi.org/10.1590/ s2176-6681/288236353

Freitas, I. A. \& Brandão, H. P. (2005). Trilhas de aprendizagem como estratégia para desenvolvimento de competências. ANPAD. Disponível em: <http://www.anpad.org.br/admin/pdf/enanpad2005gpra-0316.pdf> Acesso em 22 set. 2020.

Gamboa, S. S. (1998). Epistemologia da Pesquisa em Educação. Praxis,

Garcia, A. D. C., Dorsa, A. C., Oliveira, E. D. \& Castilho, M. A. (2018). A educação profissional no Brasil: origem e trajetória. Revista Vozes dos Vales, 12, 1-18.

Gil, A. C. (2002). Como elaborar Projetos de Pesquisa. 4a ed. Atlas.

Goergen, P. (2005). Educação e valores no mundo contemporâneo. Educação \& Sociedade, 26(92), 983-1011. https://doi.org/10.1590/S0101-73302005000300013

Lakatos, E. M. \& Marcori, M. A. (1992). Metodologia do trabalho científico: procedimentos básicos, pesquisa bibliográfica, projeto e relatório, publicações e trabalhos científicos. 4. ed. Atlas.

McClelland, D. C. (1973). Testing for competence rather than for "intelligence". The American Psychologist, 28(1), 1-14. https://doi.org/10.1037/h0034092 
Moura, D. (2007). Educação Básica e Educação Profissional e Tecnologia: dualidade histórica e perspectivas de integração. HOLOS, 2, 4-30. doi:https://doi.org/10.15628/holos.2007.11

Perrenoud, P. (1999). Construir as competências desde a escola. Porto Alegre: Artmed.

Santos, M. C. C., Barra, S. R. (2012). O projeto integrador como ferramenta de construção de habilidades e competências no ensino da engenharia e tecnologia. XL Congresso Brasileiro de Educação em Engenharia - COBENGE. Disponível em http://www.abenge.org.br/cobenge/ arquivos/7/artigos/104305.pdf. Acesso em 01 set 2020.

Saviani, D. (2005). Instituições escolares: conceito, história, historiografia e práticas. Cadernos de História da Educação, 4. Recuperado de http://www.seer.ufu.br/index.php/che/article/view/382 . (2007). Trabalho e educação: fundamentos ontológicos e históricos. Revista Brasileira de Educação, 12(34), 152-165. https://doi.org/10.1590/S1413-24782007000100012

Streck, D. R. \& Viola, S. E. A. (2014). O ethos de uma educação para os direitos humanos. In: Antonelli, T. S.; Brabo, M. (Orgs.). Direitos humanos, ética, trabalho e educação. Icone.

Torres, C. A., Burbules, N. (2004). Globalização e educação: uma introdução. In: Torres, C. A., Burbules, N. (Orgs.) Globalização e educação: perspectivas críticas. Artmed. 
